\title{
Bilateral trading with incomplete information and Price convergence in a small market: The continuous support case
}

\author{
Kalyan Chatterjee* Kaustav Das $^{\dagger}$
}

November 16, 2017

\begin{abstract}
Chatterjee and Das (Chatterjee,K., Das, K. 2017, Bilateral trading and incomplete information: Price convergence in a small market.) recently examined a model of a small market with two homogeneous buyers and two heterogeneous sellers with one of the sellers having private information. They show that as agents become patient enough, for any prior belief about the type of the privately informed seller, in any stationary equilibrium, prices in all transactions converge to the highest possible valuation of the informed seller. In the model, it was assumed that the privately informed seller's type is distributed on a two-point support. In this note, we argue that the asymptotic uniqueness result also holds when the privately informed seller's valuation is distributed on a continuous support. This shows the robustness of the uniqueness result obtained in Chatterjee and Das (2017).
\end{abstract}

\section{Introduction}

In a recent paper [1] we examined a model of a small market with two homogeneous buyers and two heterogeneous sellers. In that paper, it was assumed that the privately informed seller's valuation is distributed on a two-point support. Here we argue that even if this valuation has a continuous distribution on a finite support, a similar result will hold; namely as agents become patient enough, for any prior belief about the type of the privately informed

${ }^{*}$ Department of Economics, The Pennsylvania State University, University Park, Pa. 16802, USA. email; kchatterjee@psu.edu

${ }^{\dagger}$ Department of Economics, University of Exeter Business School Streatham Court, Streatham Campus, Exeter EX44ST, UK, Email: k.das@exeter.ac.uk 
seller, price offers in all stationary equilibria converge to the highest possible valuation of the privately informed seller. This shows the robustness of the result obtained in [1]. Please refer to [1] for related literature.

\section{The Environment}

We consider a small market with two uninformed homogeneous buyers and two heterogeneous sellers. Buyers $\left(B_{1}\right.$ and $\left.B_{2}\right)$ have a common valuation of $v$ for the good (the maximum willingness to pay for a unit of the indivisible good). There are two sellers. Each of the sellers owns one unit of the indivisible good. Sellers differ in their valuations. The first seller $\left(S_{M}\right)$ has a reservation value of $M$ which is commonly known. The other seller $\left(S_{I}\right)$ has a reservation value that is private information to her. The privately informed seller's valuation is continuously distributed on $(L, H]$ according to some cdf $G(s)$ where

$$
v>H>M>L=0
$$

Let $g($.$) be the density function which is assumed to be bounded:$

$$
0<\mathrm{g} \leq g(s) \leq \bar{g}
$$

We consider an infinite horizon multiplayer bargaining game with one-sided offers and discounting. The extensive form is identical to that in [1].

At any time point $t$, the buyer's posterior distribution about the privately informed seller's valuation can be characterized by a unique number $s^{e}$, which is the lowest possible valuation of the seller. With a slight abuse of terminology, we will call $s^{e}$ the buyer's posterior. We begin our analysis by discussing an important finiteness result of the two-player game with one-sided asymmetric information.

\subsection{Finiteness result of the Two-player Game}

Consider the infinite horizon bargaining game between a buyer with valuation $v$ and the seller with private information. Every period, the buyer makes the offer.

From [2] we can infer that in such a game, as the discount factor $\delta \rightarrow 1$, the price offered by the buyer converges to $H$ for any posterior of the buyer. Further, as $\delta \rightarrow 1$, the maximum number of periods for which the game would last is bounded above by $N^{*}$ where $N^{*}<\infty$. This implies that the privately informed seller by rejecting a sequence of equilibrium offers a finite number of times can get an offer of $H$. 


\subsection{Four-player game}

In this subsection, we analyze the four-player game. Analogous to the discrete types case (Chatterjee and Das (2017) [1]), we restrict our focus on stationary equilibria. We show that if there exists a stationary equilibrium in the four-player game, then the asymptotic outcome for all such equilibria is unique. That is, as $\delta \rightarrow 1$, price offers in all transactions go to $H$. We show this in the following steps.

Let $S$ be the set of posteriors such that for any posterior belonging to this set, a stationary equilibrium exists where, on the equilibrium path, both buyers offer only to the seller with private information. Let $\Gamma(S)$ be the set of such equilibria in case there are more than one. Let $\Gamma^{c}(S)$ be the set of all those stationary equilibria where on the equilibrium path both buyers do not solely make offers to the seller with private information when all four players are present. We first prove the following proposition.

Proposition 1 As the discount factor $\delta \rightarrow 1$, all price offers in any equilibrium belonging to the set $\Gamma^{c}(S)$ converge to $H$.

Proof. We begin this proposition by observing that similar to the logic applied in the corresponding lemmas of [1], it can be shown in the present case as well that for any equilibrium in $\Gamma^{c}(S)$, both buyers on the equilibrium path cannot solely make offers to $S_{M}$, and $S_{M}$ always accepts an equilibrium offer immediately. In the following lemma, we show that for any equilibrium in the set of equilibria considered, the informed seller by rejecting equilibrium offers for a finite number of periods can take the posterior to $H$.

Lemma 1 Consider an arbitrary equilibrium in the set $\Gamma^{c}(S)$. Given a s and $\delta$, there exists $a T_{s}(\delta)>0$ such that, conditional on getting offers, the informed seller can get an offer of $H$ in $T_{s}(\delta)$ periods from now by rejecting all offers she gets in between. $T_{s}(\delta)$ depends on the sequence of equilibrium offers and corresponding strategies of the responders in the candidate equilibrium. $T_{s}(\delta)$ is uniformly bounded above as $\delta \rightarrow 1$.

\section{Proof.}

In any equilibrium, a positive mass of types of the informed seller should always accept. If a particular type $s$ accepts, then all types $s^{\prime}<s$ should also accept. Thus, rejection always leads to an upward revision of the posterior. This proves the first part of the lemma.

We will show that given a posterior $s$, in any equilibrium, the mass of types of the informed seller accepting a sequence of equilibrium offers is always bounded below as $\delta \rightarrow 1$.

As argued in the discrete types case ([1]), we only need to consider the case where two buyers offer to $S_{I}$ with positive probability. For a given $s$, let $p_{l}$ be the minimum offer 
which gets accepted by a positive mass of types of $S_{I}$. We know that there exists a possible outcome such that $S_{I}$ only gets the offer of $p_{l}$. Since $S_{M}$ always accepts an equilibrium offer immediately, $S_{I}$ in such a situation knows that rejecting the offer will lead to a two-player game. By invoking the finiteness property of the two-player game, we can infer that as $\delta \rightarrow 1$, the number of rejections required for the informed seller to get an offer of $H$ converges to some $\tilde{T}(0<\tilde{T}<\infty)$. Further, the posteriors in each time period $t=1, . ., \tilde{T}-1$ reaches a limit $s_{t}$. Let $p_{t}$ be the price offered in time period $t>0$, following a rejection from the informed seller in $t-1$. Note that $p_{0}=p_{l}$ and $p_{\tilde{T}}=H$.

Suppose there exists an equilibrium of the four-player game such that $p_{l}$ is the initial price offer and the number of rejections required for the informed seller to get $H$ is not bounded above as $\delta \rightarrow 1$. This implies that we can find a $\delta^{\prime}<1$ such that for all $\delta>\delta^{\prime}$, we have $s_{t}^{u}<s_{t}$ for all $t=1,2, \ldots, \tilde{T}$. Here, $s_{t}^{u}$ is the updated posterior following a rejection in $t-1$. Let $p_{t}^{u}$ be the offer (or highest of the offers) to the informed seller at time point $t \geq 1$.

We will now show that for all $t \geq 1, p_{t}^{u}>p_{t}$.

Consider $t=1$. We know that

$$
p_{l}-s_{1}=\delta\left(p_{1}-s_{1}\right) \Rightarrow \delta p_{1}=p_{l}-(1-\delta) s_{1}
$$

Similarly,

$$
p_{l}-s_{1}^{u}=\delta\left(p_{1}^{u}-s_{1}^{u}\right) \Rightarrow \delta p_{1}^{u}=p_{l}-(1-\delta) s_{1}^{u}
$$

Thus, we have $\delta\left(p_{1}^{u}-p_{1}\right)=(1-\delta)\left(s_{1}-s_{1}^{u}\right)>0 \Rightarrow p_{1}^{u}>p_{1}$.

Consider $t>1$. We will show that if $p_{t-1}^{u}>p_{t-1}$, then $p_{t}^{u}>p_{t}$.

$$
p_{t-1}-s_{t}=\delta\left(p_{t}-s_{t}\right) \Rightarrow \delta p_{t}=p_{t-1}-(1-\delta) s_{t}
$$

Similarly,

$$
p_{t-1}-s_{t}^{u}=\delta\left(p_{t}^{u}-s_{t}^{u}\right) \Rightarrow \delta p_{t}^{u}=p_{t-1}^{u}-(1-\delta) s_{t}^{u}
$$

Thus, we have $\delta\left(p_{t}^{u}-p_{t}\right)=p_{t-1}^{u}-p_{t-1}+(1-\delta)\left(s_{t}-s_{t}^{u}\right)>0$. Hence for all $t \geq 1, p_{t}^{u}>p_{t}$.

This implies that $p_{\tilde{T}} \geq H$. Hence, the price offer reaches $H$ after a finite number of rejections. This is contradictory to our conjectured hypothesis. Thus, in any equilibrium, the informed seller can get an offer of $H$ after rejecting for a finite number of times. This concludes the proof of the lemma.

The above lemma shows that in any equilibrium belonging to the set $\Gamma^{c}(S)$, the privately informed seller can get an offer of $H$ by rejecting equilibrium offers a finite number of times. Similar to the logic used in applied in [1] for the corresponding lemmas, we can argue that for any equilibrium in $\Gamma^{c}(S)$ both buyers cannot solely make offer to $S_{I}$ and both buyers make 
offers to $S_{M}$ with positive probability. Finally, similar to [1] we can show that as $\delta \rightarrow 1$, for any equilibrium in $\Gamma^{c}(S)$, all price offers converge to $H$. This concludes the proof of the proposition.

We will now show that the set $S$ is empty.

Lemma 2 Let $S$ be the set of all posteriors $(s<H)$ such that for $s \in S$, it is possible to have a stationary equilibrium where both buyers offer only to $S_{I}$. The set $S$ is empty

Proof. Suppose $S$ is non-empty. This implies that for any $s \in S$, it is possible to have a stationary equilibrium such that both buyers on the equilibrium path offer only to $S_{I}$. Since a positive mass of types of $S_{I}$ always accepts an offer in equilibrium, next period $S_{M}$ will face a two-player game with a positive probability. This implies that the expected continuation payoff of $S_{M}$ is strictly less than $H$. Hence, the highest price offer in such an equilibrium is always strictly less than $H$.

Let $\bar{p}$ be the largest price offer (as argued $\bar{p}<H$ ), for any $s$ in such an equilibrium (W.L.O.G, such an offer exists). In any equilibrium, a positive mass of types of the informed seller always accept an equilibrium offer. From Bayes' Theorem and $\delta<1$, we can infer that the sequence of prices must be increasing. Consider the offer of $\bar{p}$. In the continuation game, given the updated belief conditional on the offer being rejected, the equilibrium will either be in $\Gamma(S)$ or in $\Gamma^{c}(S)$. In the former case any type $s^{\prime}<H$ accepts this offer with probability 1. This means in the continuation game by Bayes' rule we will have the posterior to be equal to $H$ and hence, the price offer will be equal to $H$. Hence, all these $s$ 'types can profitably deviate for high values of $\delta$. In the latter case, there exists a type $s^{\prime}<H$ who accepts this offer. In the previous proposition, we have argued that for high values of $\delta$ all price offers converge to $H$. This implies the type $s$ ' which accepts the offer can profitably deviate for high values of $\delta$. Hence, we cannot have $S$ to be non-empty. This concludes the proof.

Since $S$ is empty, $\Gamma^{c}(S)$ is the set of all stationary. We now state our main result of this note in the theorem below,

Theorem 1 In any arbitrary stationary equilibrium of the four-player game, as the discount factor goes to 1 , price offers in all transactions converge to $H$ for all values $s \in[L, H)$.

Proof. The proof the theorem follows directly from proposition (1) and lemma (2). 


\section{Conclusion}

We have shown that even when the privately informed seller's valuation has a continuous distribution on a finite support, the asymptotic equilibrium outcome in the model of a small market is unique. This result might help us to make a more tractable analysis of the small market with more than one privately informed sellers.

\section{Acknowledgements}

The authors wish to thank Siddhartha Bandopadhyay, Martin Cripps, Bhaskar Dutta, Faruk Gul, Ed Green, Vijay Krishna, Selçuk Özyurt, Larry Samuelson and Asher Wolinsky for their insightful comments and suggestions. We also thank the conference participants of the Royal Economic Society, World Congress of the Econometric Society and the seminar participants at the Brown University and the Indian Statistical Institute for helpful comments. Finally, we thank the editor Roberto Serrano for his suggestions. Dr Chatterjee would also like to thank the Institute for Advanced Study, Princeton, and the Richard B. Fisher endowment for financial support of his membership of the Institute during the year 2014-15.

\section{References}

[1] Chatterjee, K., Das, K. 2017. "Bilateral trading and incomplete information: Price convergence in a small market", Games and Economic Behavior, Vol. 106 (November 2017), Pages: 89-113.

[2] Fudenberg, D., Levine, D., and Tirole, J. , 1985. "Infinite-Horizon Models of Bargaining with One-Sided Incomplete Information. "A. Roth (ed.), Game-Theoretic Models of Bargaining , Cambridge University Press . 